\title{
MRI Evaluation of Suspected Pathologic Fracture at the Extremities from Metastasis: Diagnostic Value of Added Diffusion-Weighted Imaging
}

\author{
Sun-Young Park, MD, $\mathrm{PhD}^{1,2}$, Min Hee Lee, $M D, \mathrm{PhD}^{1}$, Ji Young Jeon, $\mathrm{MD}^{3}$, Hye Won Chung, MD, $\mathrm{PhD}^{1}$, \\ Sang Hoon Lee, MD, $\mathrm{PhD}^{1}$, Myung Jin Shin, MD, $\mathrm{PhD}^{1}$

\begin{abstract}
${ }^{1}$ Department of Radiology and Research Institute of Radiology, University of Ulsan College of Medicine, Asan Medical Center, Seoul, Korea; ${ }^{2}$ Department of Radiology, Hallym University Sacred Heart Hospital, Anyang, Korea; ${ }^{3}$ Department of Radiology, Gil Medical Center, Gachon
\end{abstract} \\ University College of Medicine, Incheon, Korea
}

Objective: To assess the diagnostic value of combining diffusion-weighted imaging (DWI) with conventional magnetic resonance imaging (MRI) for differentiating between pathologic and traumatic fractures at extremities from metastasis.

Materials and Methods: Institutional Review Board approved this retrospective study and informed consent was waived. This study included 49 patients each with pathologic and traumatic fractures at extremities. The patients underwent conventional MRI combined with DWI. For qualitative analysis, two radiologists (R1 and R2) independently reviewed three imaging sets with a crossover design using a 5-point scale and a 3-scale confidence level: DWI plus non-enhanced MRI (NEMR; DW set), NEMR plus contrast-enhanced fat-saturated T1-weighted imaging (CEFST1; CE set), and DWI plus NEMR plus CEFST1 (combined set). McNemar's test was used to compare the diagnostic performances among three sets and perform subgroup analyses (single vs. multiple bone abnormality, absence/presence of extra-osseous mass, and bone enhancement at fracture margin).

Results: Compared to the CE set, the combined set showed improved diagnostic accuracy (R1, 84.7 vs. 95.9\%; R2, 91.8 vs. $95.9 \%, p<0.05)$ and specificity (R1, $71.4 \%$ vs. $93.9 \%, p<0.005 ; \mathrm{R} 2,85.7 \%$ vs. $98 \%, p=0.07$ ), with no difference in sensitivities $(p>0.05)$. In cases of absent extra-osseous soft tissue mass and present fracture site enhancement, the combined set showed improved accuracy (R1, 82.9-84.4\% vs. 95.6-96.3\%, $p<0.05$; R2, 90.2-91.1\% vs. 95.1-95.6\%, $p<$ 0.05 ) and specificity (R1, 68.3-72.9\% vs. $92.7-95.8 \%, p<0.005 ; \mathrm{R} 2,83.0-85.4 \%$ vs. $97.6-98.0 \%, p=0.07)$.

Conclusion: Combining DWI with conventional MRI improved the diagnostic accuracy and specificity while retaining sensitivity for differentiating between pathologic and traumatic fractures from metastasis at extremities.

Keywords: Functional MRI; Diffusion; Fracture; Neoplasm metastasis; Extremities

\section{INTRODUCTION}

Pathologic fractures of the long bones occur in $17-43 \%$ of patients with bone metastasis from lung cancer or other solid tumors (1). This is a devastating skeletal complication of advanced malignancies, which occasionally occurs as the first manifestation of cancer. Accurate diagnosis of pathologic fractures in cancer patients is crucial because the appropriate treatment options could differ from those for traumatic fractures, thus affecting the quality of life during an extended survival period (2).

Although plain radiography is the primary screening technique for evaluating suspected pathologic fractures, conventional magnetic resonance imaging (MRI) is the most

Received August 10, 2018; accepted after revision December 4, 2018.

Corresponding author: Min Hee Lee, MD, PhD, Department of Radiology and Research Institute of Radiology, University of Ulsan College of Medicine, Asan Medical Center, 88 Olympic-ro 43-gil, Songpa-gu, Seoul 05505, Korea.

- Tel: (822) 3010-5985•Fax: (822) 476-4719•E-mail: mhlee625@gmail.com

This is an Open Access article distributed under the terms of the Creative Commons Attribution Non-Commercial License (https:// creativecommons.org/licenses/by-nc/4.0) which permits unrestricted non-commercial use, distribution, and reproduction in any medium, provided the original work is properly cited. 
preferred modality as it demonstrates superior resolution in bone marrow lesion; gadolinium-based contrast media can be recommended in order to optimize the detection and characterization of bone marrow lesion $(3,4)$. Nonetheless, diagnostic challenges remain, particularly in acute-stage fractures, which often show aggressive appearances on MRI that can hide underlying metastatic lesions.

Diffusion-weighed imaging (DWI) is a functional imaging technique that is based on differences in the Brownian motion of water molecules between tissues. Various studies have been published on the utility of DWI in the detection, characterization, and longitudinal evaluation of bone and soft tissue tumors (5-7). In previous studies, although fractures have been evaluated with DWI, the focus has mostly been on differentiating malignant from osteoporotic vertebral compression fractures through measurement of apparent diffusion coefficient (ADC) values (8-13). The usefulness of DWI in evaluating suspected pathologic fractures at extremities has not yet been elucidated. Furthermore, no published data within the scope of the authors' knowledge, are available on how addition of DWI to conventional MRI contributes to diagnostic value. Therefore, our study aimed to assess the diagnostic value of combining DWI with conventional MRI in differentiating between pathologic and traumatic fractures at extremities from metastasis.

\section{MATERIALS AND METHODS}

\section{Patients}

This study was approved by the Institutional Review Board of our hospital, which waived requirement for

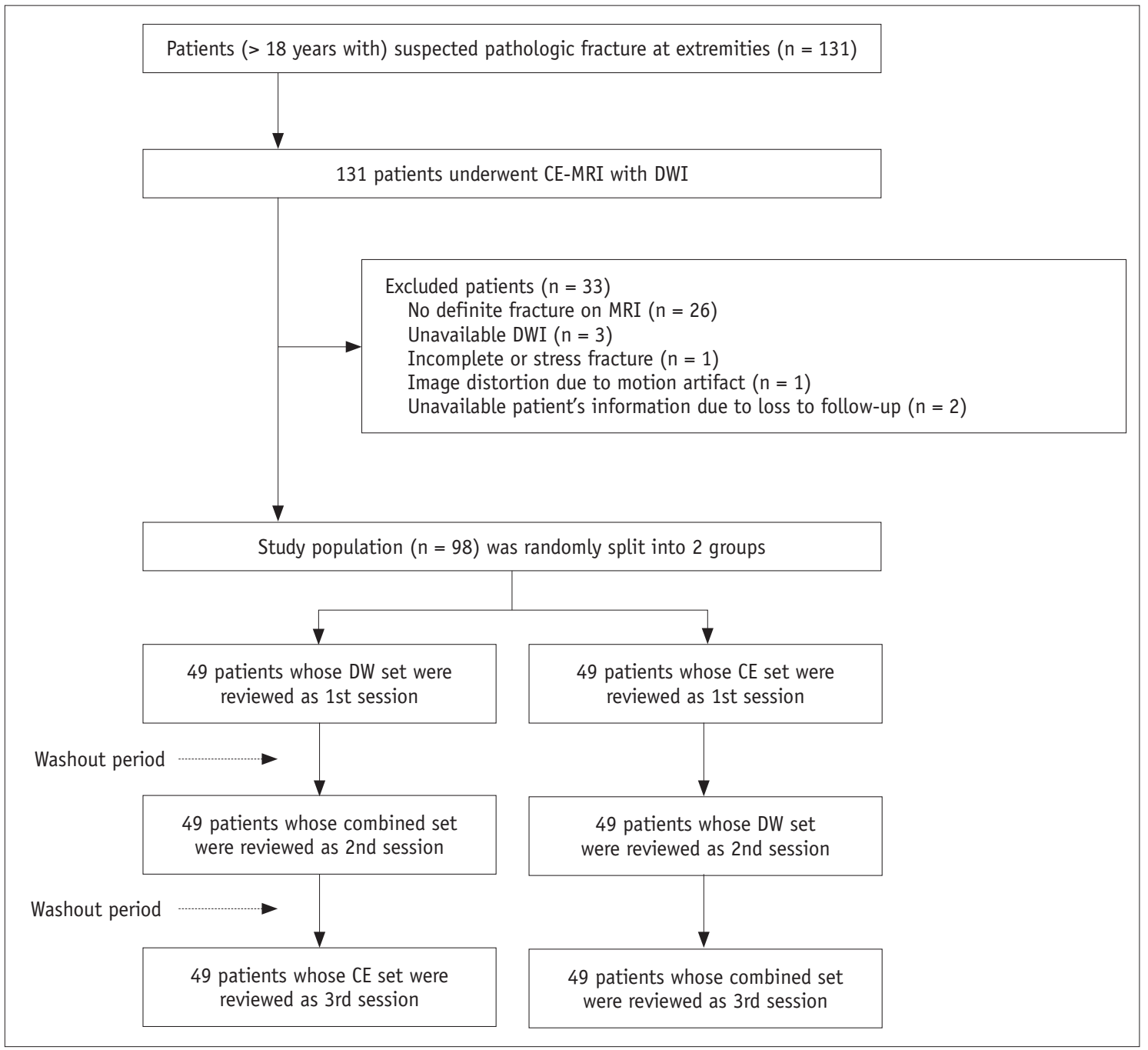

Fig. 1. Study flowchart. $C E=$ contrast-enhanced, $C E$ set $=$ NEMR plus CE fat-saturated T1-weighted imaging, Combined set $=$ DWI plus CE set, DWI = diffusion-weighted imaging, DW set $=$ DWI plus NEMR, MRI = magnetic resonance imaging, NEMR = non-enhanced MRI 
informed consent because of the retrospective nature of the study. From September 2013 to December 2016, a total of 131 patients who presented with suspected pathologic fracture at extremities on initial plain radiographs obtained in the emergency room or at an outpatient clinic were enrolled; they had subsequently undergone contrastenhanced (CE) MRI with DWI. Excluded criteria were age $\leq 18$ years or pathologic fractures due to primary bone tumors. Some patients were excluded because of the following: a possible fracture on initial plain radiographs that was not evident on MRI $(n=26)$; unavailable DWI ( $n$ $=3)$; incomplete or stress fracture on MRI $(n=1)$; image distortion due to motion artifact $(n=1)$; loss to follow-up ( $n$ $=2$ ). Finally, 98 patients were analyzed (Fig. 1). The final diagnosis of a pathologic fracture was made by pathologic confirmation $(n=59)$ or inspection of the fracture site during surgery $(n=8)$ and by clinical and imaging follow-up in patients who were not considered appropriate candidates for surgery owing to their general conditions or short life expectancy $(n=31)$. of 98 subjects, 49 had pathologic and 49 had traumatic fractures; eighty-eight $(89.8 \%)$ patients had an underlying primary malignancy, absence of which was confirmed after diagnostic imaging workup and follow- up examinations including chest and abdominopelvic CT and/or positron emission tomography-СT. Table 1 shows a comparison of the demographic data, including underlying primary malignancies and fracture locations at extremities, between the groups. Most patients had a history of trauma in the spectrum of low-to-high-energy trauma. The mean interval between the onset of symptoms and MRI was 12.2 days (range, 0-149 days; median, 1 day).

\section{Conventional and Diffusion-Weighted MRI}

MRI was performed using different MRI scanners with dedicated coils. A 1.5T MAGNETOM Avanto (Siemens Healthineers, Erlangen, Germany) MRI scanner was used in 62 patients, and 3T MRI scanners were used in 36 patients: Achieva (Philips Healthcare, Best, The Netherlands) in 23, Ingenia (Philips Healthcare) in nine, and MAGNETOM Skyra (Siemens Healthineers) in four. Conventional MRI sequences obtained were as follows: non-enhanced MRI (NEMR) including axial, sagittal, or coronal T1-weighted imaging (T1WI), and axial, sagittal, or coronal T2-weighted imaging (T2WI) with/without fat-saturation, as well as CE fat-saturated T1-weighted imaging (CEFST1) for all three planes. Before intravenous contrast material injection,

Table 1. Characteristics of Study Population

\begin{tabular}{|c|c|c|}
\hline Patients Included $(n=98)$ & Patients with Pathologic Fractures $(n=49)$ & Patients with Traumatic Fractures $(n=49)$ \\
\hline Age (years $\pm S D)$ & $64 \pm 12$ & $66 \pm 14$ \\
\hline Sex (male/female) & $19 / 30$ & $21 / 29$ \\
\hline \multicolumn{3}{|l|}{ Underlying primary malignancy $(n=88)$} \\
\hline Lung cancer & 13 & 8 \\
\hline Hepatocellular carcinoma & 9 & 3 \\
\hline Breast cancer & 5 & 4 \\
\hline Colorectal cancer & 4 & 5 \\
\hline Advanced gastric cancer & 0 & 3 \\
\hline Cholangiocarcinoma & 3 & 0 \\
\hline Prostate cancer & 3 & 1 \\
\hline Renal cell carcinoma & 1 & 2 \\
\hline Cervical cancer & 1 & 1 \\
\hline Thyroid cancer & 1 & 3 \\
\hline 0thers* & 9 & 9 \\
\hline Absent primary malignancy $(n=10)$ & 0 & 10 \\
\hline \multicolumn{3}{|l|}{ Fracture location $(n=98)$} \\
\hline Femur & 33 & 41 \\
\hline Humerus & 14 & 5 \\
\hline 0thers ${ }^{\dagger}$ & 2 & 3 \\
\hline
\end{tabular}

*Involved combined hepatocellular-cholangiocarcinoma $(n=1)$, sinonasal undifferentiated carcinoma in nasal cavity ( $n=1)$, gastrointestinal stromal tumor $(n=2)$, undifferentiated pleomorphic sarcoma $(n=1)$, esophageal cancer $(n=1)$, vaginal cancer $(n=$ $1)$, multiple myeloma $(n=4)$, lymphoma $(n=1)$, pancreatic cancer $(n=2)$, ovary cancer $(n=1)$, leiomyosarcoma $(n=1)$, primitive neuroectodermal tumor $(n=1)$, 'Involved clavicle $(n=2)$, tibia $(n=2)$, scapula $(n=1) . S D=$ standard deviation 
DWI was performed in the axial plane using a spin-echo, single-shot echo-planar imaging sequence. $A D C$ maps were calculated from $b=0,400$, and 1400 images. Acquisition parameters were adapted to the anatomical regions under assessment. Table 2 summarizes the detailed parameters for each MRI sequence.

\section{Imaging Analysis}

Before the image review, an independent reader (with 4 years of experience in musculoskeletal imaging) was asked to evaluate conventional MRI without clinical and pathologic findings. First, the presence/absence of a focal bone abnormality at the fracture site was determined. The maximum diameter of the focal bone abnormality was recorded with the number of focal bone lesions as single or multiple, when a lesion was present within any field-ofview (FOV) of the three planes. A focal bone abnormality was defined as an abnormal bone marrow signal lesion with discrete margin at and/or around a fracture site on T1WI and/or discrete enhancement on CEFST1. Second, the presence of an extra-osseous soft tissue component was determined. Third, the presence of bone marrow enhancement at the fracture margin was evaluated on one or both sides of the fracture margin. We differentiated imaging findings of bone marrow edema from the focal bone abnormality based on an ill-defined, moderately decreased signal intensity on T1WI without a discrete enhanced margin on CEFST1.

For the image review, three imaging sets were defined: DWI plus NEMR (DW set), NEMR plus CEFST1 (CE set), and DWI plus NEMR plus CEFST1 (combined set). To differentiate between pathologic and traumatic fractures, a qualitative analysis was performed for each imaging set using a 5-point scale as follows: grade 1, definite traumatic fracture; grade 2, probable traumatic fracture; grade 3, possible pathologic fracture; grade 4, probable pathologic fracture; and grade 5, definite pathologic fracture. Subsequently, the scores on the 5-point scale were regrouped into a 3-level confidence system, with 1 indicating the lowest confidence and 3 the highest confidence. When evaluating DW and combined sets, DW-MR images and $A D C$ map were placed alongside and evaluated for the presence of a focal bone abnormality at or around the fracture site on conventional MRI with a high signal on DWI and impeded water diffusivity on ADC map; this approach was similar to the method used by Sung et al. (10).

Two reviewers (one with 3 and the other with 19 years of experience in musculoskeletal imaging), who were not involved in patient selection, participated in the examination process retrospectively and independently. Three review sessions using a crossover design were performed by the two reviewers. In each session, images were randomly assigned to two anonymous groups by the previously mentioned independent reader. For three review sessions, three different combinations were created by combining two of the 3 groups of image sets, that is, the $D W, C E$, and combined sets. During the first session, the DW and CE sets in each group were evaluated. The combined and DW sets during the second session, and the CE and

Table 2. MRI Sequence Parameters

\begin{tabular}{|c|c|c|}
\hline Parameters & Standard Imaging & DWI \\
\hline \multirow{4}{*}{ Repetition time (ms)/echo time (ms) } & T1-weighted coronal or sagittal imaging: 456-587/12-20 & $5000-5200 / 61-85$ \\
\hline & T2-weighted coronal or sagittal imaging: $2291-2978 / 60-80$ & \\
\hline & T1-weighted axial imaging: $475-588 / 15$ & \\
\hline & T2-weighted axial imaging: 2291-2696/80 & \\
\hline Fat suppression & SPAIR & SPAIR \\
\hline Echoplanar imaging factor & NA & 67 \\
\hline Matrix & $325 \times 257-448 \times 250$ & $184 \times 256$ \\
\hline \multirow{3}{*}{ Field of view $(\mathrm{mm})$} & Sagital imaging: 100-400 & $179-380$ \\
\hline & Axial imaging: 80-399 & \\
\hline & Coronal imaging: $198-449$ & \\
\hline Section thickness (mm) & $1-3$ & $2.5-5$ \\
\hline Intersection gap (mm) & $0-0.05$ & $0-1$ \\
\hline \multirow{2}{*}{ Turbo factor } & T1-weighted imaging: 3 & NA \\
\hline & T2-weighted imaging: 16 & \\
\hline Number of signals acquired & $2-4$ & $2-6$ \\
\hline
\end{tabular}

DWI = diffusion-weighted imaging, MRI = magnetic resonance imaging, NA = not applicable, SPAIR = spectral attenuated inversion recovery 
Table 3. Analysis Results of Conventional MRI Findings at Fracture Margins

\begin{tabular}{|c|c|c|c|}
\hline Imaging Findings & Pathologic Fractures $(n=49)$ & Traumatic Fractures $(n=49)$ & Total $(n=98)$ \\
\hline \multicolumn{4}{|l|}{ Focal bone abnormality } \\
\hline Presence (single/multiple) & $46(16 / 30)$ & $11(9 / 2)$ & $57(25 / 32)$ \\
\hline Absence & 3 & 38 & 41 \\
\hline \multicolumn{4}{|c|}{ Extra-osseous soft tissue component } \\
\hline Presence & 15 & 1 & 16 \\
\hline Absence & 34 & 48 & 82 \\
\hline \multicolumn{4}{|l|}{ Fracture site enhancement } \\
\hline Presence & 49 & 41 & 90 \\
\hline Absence & 0 & 8 & 8 \\
\hline
\end{tabular}

combined sets during the third session were evaluated in the same manner (Fig. 1). To reduce recall bias, each session was conducted 4 weeks apart. All pathologic results and clinical and imaging data were reviewed thoroughly by the independent reader after images were reviewed.

\section{Statistical Analysis}

The sensitivity, specificity, and accuracy of three sets were calculated for differentiating between pathologic and traumatic fractures. Subgroup analyses according to the presence of focal bone abnormality and the number of bone lesions within FOV, absence of extra-osseous soft tissue component, and presence of bone enhancement at fracture margins on conventional MRI was performed for calculating the sensitivity, specificity, and accuracy for each subgroup. McNemar's test was used to analyze the differences in diagnostic performance among three image sets in all patients and subgroups. Inter-reviewer agreement was tested using weighted $\kappa$ statistics. A $\kappa$ value of $0.00-0.20$ indicated slight agreement; 0.21-0.40, fair agreement; 0.41-0.60, moderate agreement; $0.61-0.80$, good agreement; and 0.81-1.00, excellent agreement. Statistical significance was set at $p$ value $<0.05$. Commercially available software (MedCalc 17.0; MedCalc Software, Mariakerke, Belgium, and SPSS 24.0; IBM Corp., Armonk, NY, USA) were used for statistical analyses.

\section{RESULTS}

\section{Conventional MRI Findings at Fracture Margins with}

\section{Pathologic Correlation}

MRI revealed focal bone abnormalities in 57 (58.2\%) of the 98 patients; 46 pathologic and 11 traumatic fractures were observed in these patients. Three pathologic fractures without a focal bone abnormality identified by MRI had diffuse bone marrow signal changes ( $n=$ 2 ) and a hematoma at the fracture site $(n=1)$. Eleven traumatic fractures showing focal bone abnormalities were confirmed as "no tumor present" during surgery $(n=9)$ and by clinical and radiological follow-up $(n=2)$; three of these had pathologically confirmed hematopoietic marrow, intramedullary hematoma, and hematoma with fat necrosis. The median maximum diameter of the focal bone abnormalities was $5.8 \mathrm{~cm}$ (range, $0.5-22 \mathrm{~cm}$ ) in pathologic fractures and $3.2 \mathrm{~cm}$ (range, $1-11.4 \mathrm{~cm}$ ) in traumatic fractures. Among the 57 patients with focal bone abnormalities, single lesions were found in 25 (43.9\%) patients (16 pathologic and nine traumatic fractures) and multiple lesions in $32(56.1 \%$ ) patients (30 pathologic and two traumatic fractures). Two traumatic cases had multiple metastases in the pelvic bones; however, femoral fractures were confirmed as traumatic after operation.

An extra-osseous soft tissue component was observed in $16(16.3 \%)$ patients (15 pathologic and one traumatic fracture). The lesion of one traumatic fracture was confirmed as non-union with granulation tissue.

Bone enhancement at fracture margins was present in 90 (91.8\%) patients; 49 (100\%) pathologic and 41(83.7\%) traumatic fractures were observed in these patients (Table 3 ).

\section{Diagnostic Performance for Differentiation between Pathologic and Traumatic Fractures}

Table 4 lists the sensitivity, specificity, and accuracy for the diagnosis of pathologic fracture at extremities in the DW, CE, and combined sets using 5-point scale, for each reader.

Diagnostic accuracy was the highest for combined set for both readers (reader 1 and 2, 95.9\%). For reader 1 , compared to the CE set, the combined set showed significantly improved diagnostic accuracy $(84.7 \%$ vs. 
Table 4. Diagnostic Performance in Overall and Subgroup Analysis for Evaluating Pathologic Fractures in Two Readers Using 5-Point Scale

\begin{tabular}{|c|c|c|c|c|c|c|}
\hline & \multirow{2}{*}{ CE set $(\%)$} & \multirow{2}{*}{ DW set $(\%)$} & \multirow{2}{*}{ Combined set $(\%)$} & \multicolumn{3}{|c|}{$P$} \\
\hline & & & & CE set vs. DW set & DW set vs. Combined set & CE set vs. Combined set \\
\hline \multicolumn{7}{|l|}{ Overall $(n=98)$} \\
\hline \multicolumn{7}{|l|}{ Reader 1} \\
\hline Sensitivity & 98.0 & 93.9 & 98.0 & 0.63 & 0.5 & 1.0 \\
\hline Specificity & 71.4 & 81.6 & 93.9 & 0.36 & 0.07 & $0.001^{*}$ \\
\hline Accuracy & 84.7 & 87.8 & 95.9 & 0.21 & 0.34 & $0.003^{*}$ \\
\hline \multicolumn{7}{|l|}{ Reader 2} \\
\hline Sensitivity & 98.0 & 95.9 & 94.9 & 1.00 & 1.00 & 0.63 \\
\hline Specificity & 85.7 & 89.8 & 98.0 & 0.73 & 0.13 & 0.07 \\
\hline Accuracy & 91.8 & 92.9 & 95.9 & 0.55 & 0.13 & $0.04^{*}$ \\
\hline \multicolumn{7}{|c|}{ Single bone abnormality $(n=25)$} \\
\hline \multicolumn{7}{|c|}{ Reader 1} \\
\hline Sensitivity & 93.7 & 93.8 & 100.0 & 1.0 & 1.0 & 1.0 \\
\hline Specificity & 44.4 & 77.8 & 77.8 & 0.38 & 1.0 & 0.25 \\
\hline Accuracy & 76.0 & 88.0 & 92.0 & 0.45 & 1.0 & 0.63 \\
\hline \multicolumn{7}{|l|}{ Reader 2} \\
\hline Sensitivity & 93.8 & 93.8 & 100.0 & 1.0 & 1.0 & 1.0 \\
\hline Specificity & 77.8 & 88.9 & 100.0 & 1.0 & 1.0 & 0.5 \\
\hline Accuracy & 88.0 & 92.0 & 100.0 & 1.0 & 1.0 & 1.0 \\
\hline \multicolumn{7}{|c|}{ Absent extra-osseous soft tissue component $(n=82)$} \\
\hline \multicolumn{7}{|l|}{ Reader 1} \\
\hline Sensitivity & 97.1 & 91.2 & 97.1 & 0.63 & 0.5 & 1.0 \\
\hline Specificity & 72.9 & 83.3 & 95.8 & 0.36 & 0.07 & $0.001^{*}$ \\
\hline Accuracy & 82.9 & 86.6 & 96.3 & 0.21 & 0.34 & $0.003^{*}$ \\
\hline \multicolumn{7}{|l|}{ Reader 2} \\
\hline Sensitivity & 97.1 & 94.1 & 91.2 & 1.0 & 1.0 & 0.63 \\
\hline Specificity & 85.4 & 89.6 & 98.0 & 0.73 & 0.13 & 0.07 \\
\hline Accuracy & 90.2 & 91.5 & 95.1 & 0.55 & 0.13 & $0.039 *$ \\
\hline \multicolumn{7}{|c|}{ Present fracture site enhancement $(n=90)$} \\
\hline \multicolumn{7}{|c|}{ Reader 1} \\
\hline Sensitivity & 98.0 & 93.9 & 98.0 & 0.63 & 0.5 & 1.0 \\
\hline Specificity & 68.3 & 80.5 & 92.7 & 0.33 & 0.13 & $0.002^{*}$ \\
\hline Accuracy & 84.4 & 87.8 & 95.6 & 0.19 & 0.51 & $0.006^{*}$ \\
\hline \multicolumn{7}{|l|}{ Reader 2} \\
\hline Sensitivity & 98.0 & 95.9 & 93.9 & 1.0 & 1.0 & 0.63 \\
\hline Specificity & 83.0 & 90.2 & 97.6 & 0.45 & 0.25 & 0.07 \\
\hline Accuracy & 91.1 & 93.3 & 95.6 & 0.34 & 0.22 & 0.04 * \\
\hline
\end{tabular}

*Indicated significant difference between two groups. CEFST1 = contrast-enhanced fat-saturated T1-weighted imaging, CE set = NEMR plus CEFST1, Combined set = DWI plus NEMR plus CEFST1, DW set = DWI plus NEMR, NEMR = non-enhanced MRI

$95.9 \%, p<0.005)$ and specificity $(71.4 \%$ vs. $93.9 \%, p<$ $0.005)$. For reader 2, accuracy was increased significantly in the combined set compared to the CE set $(91.8 \%$ vs. $95.9 \%$, $p=0.04)$, with a trend of improving specificity ( $85.7 \%$ vs. $98 \%, p=0.07)$. Sensitivity was not significantly different between the combined and CE sets for any reader (both, $p>$ 0.05). Figures 2 and 3 show representative cases.

The diagnostic performance was not significantly different between the DW and CE sets and between the DW and combined sets for both readers $(p>0.05)$.

Diagnostic Performance in Subgroup Analysis according to Conventional MRI Findings

For reader 1, CE set showed accuracy (76.0-84.4\%) and moderate specificity (44.4-72.9\%) in cases of a single bone abnormality, absent extra-osseous soft tissue 

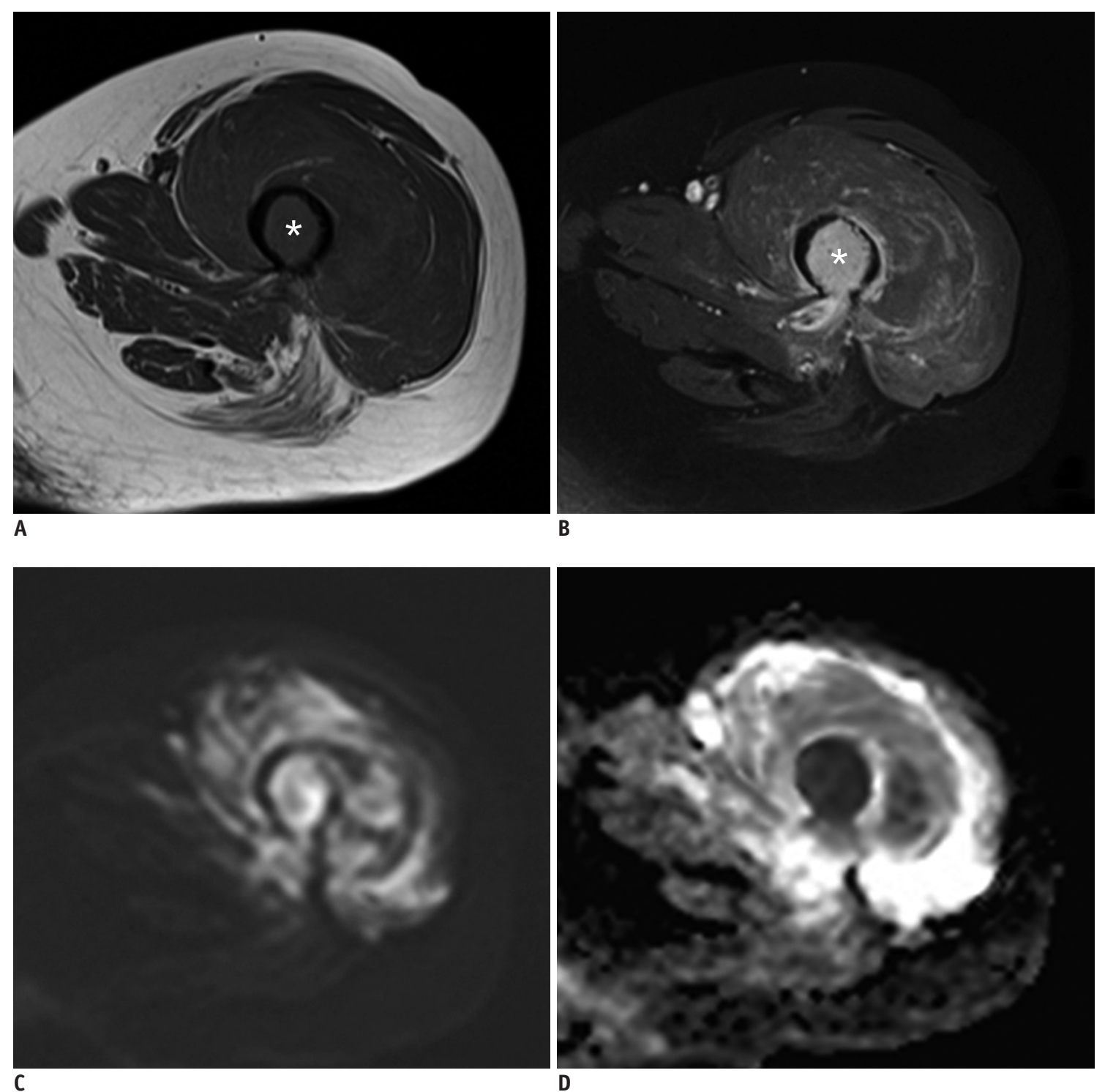

Fig. 2. CE and combined sets demonstrate correct diagnosis of pathologic fracture by both readers at left femur in 55-year-old woman with multiple myeloma.

(A) Axial T1-weighted, and (B) axial contrast-enhanced fat-saturated T1-weighted images show intramedullary enhancing single bone abnormality at fracture site with perilesional edema (asterisks). (C) Corresponding axial DWI shows high signal change at fracture site $(b=1400$ $\left.\mathrm{s} / \mathrm{mm}^{2}\right)$. (D) Axial $A D C$ map shows impeded water diffusivity. $A D C=$ apparent diffusion coefficients

component, and present bone enhancement at fracture margins. However, compared to the CE set, the combined set showed significantly improved accuracy $(82.9 \%$ vs. $96.3 \%, 84.4 \%$ vs. $95.6 \%, p<0.05)$ and specificity $(72.9 \%$ vs. $95.8 \%, 68.3 \%$ vs. $92.7 \%, p<0.005)$ in cases of absent extra-osseous soft tissue component and present bone enhancement at fracture margins.

For reader 2, in cases of absent extra-osseous soft tissue component and present bone enhancement at fracture margins, accuracy was significantly increased in the combined set compared with that in the CE set $(90.2 \%$ vs. $95.1 \%, 91.1 \%$ vs. $95.6 \%, p<0.05)$ with a trend of improvement in specificity ( $85.4 \%$ vs. $98.0 \%, 83.0 \%$ vs. $97.6 \%, p=0.07)$.

Compared to the CE set, the combined set also showed improved accuracy (R1, $76 \%$ vs. $92.0 \%$; R2, $88.0 \%$ vs. $100 \%$ ) and specificity (R1, $44.4 \%$ vs. $77.8 \% ; R 2,77.8 \%$ vs. $100 \%$ ) in cases of a single bone abnormality, but without a significant difference $(p>0.05)$ (Table 4).

The diagnostic performance was not significantly different between the DW and CE sets and between the DW and combined sets for both readers $(p>0.05)$ (Table 4$)$. 

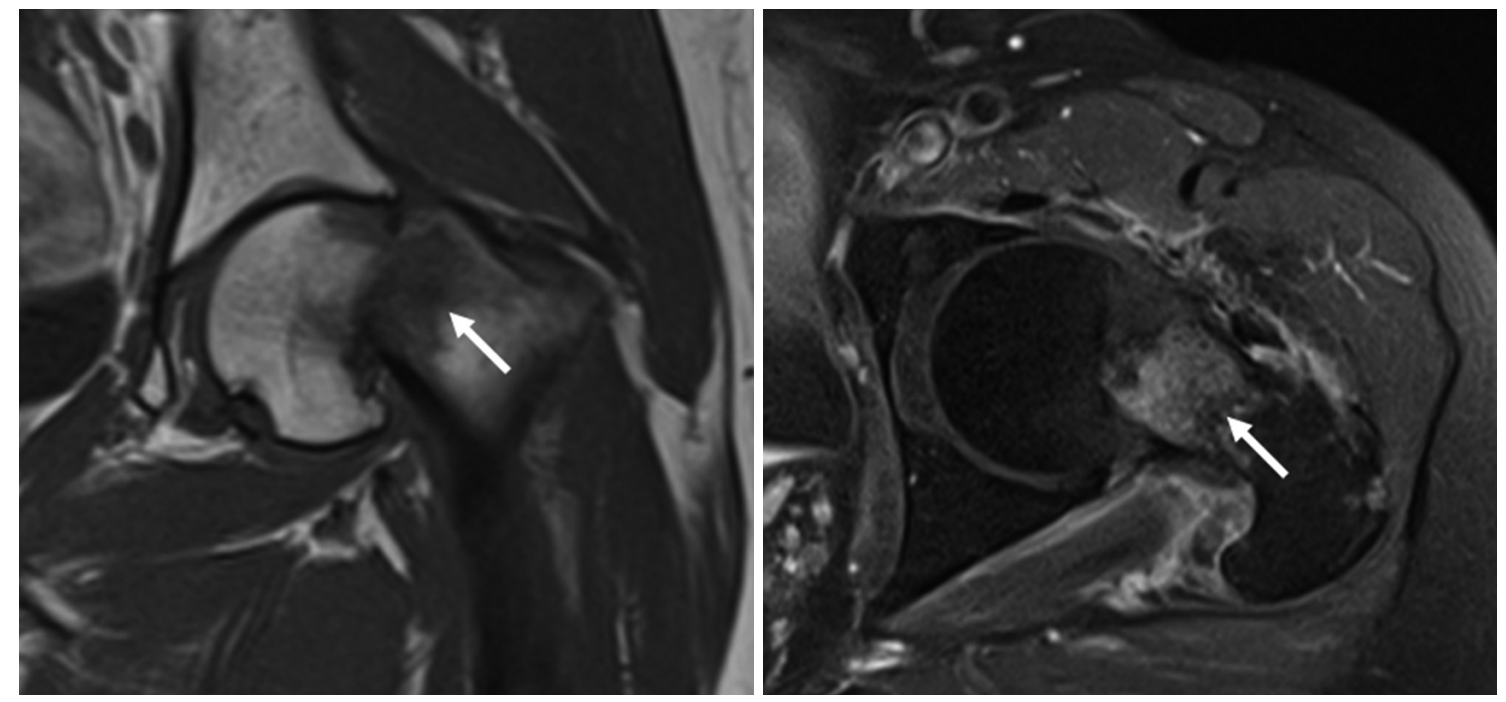

A

B
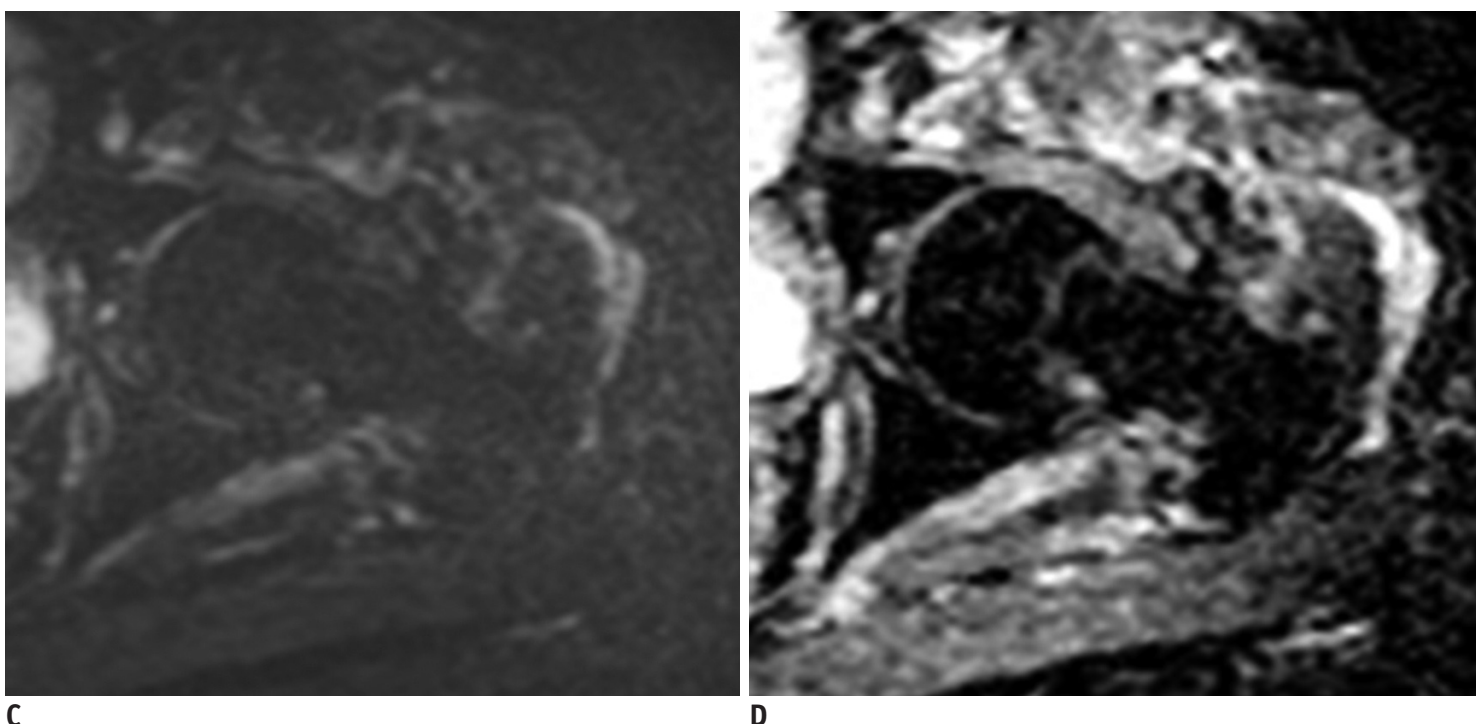

D

Fig. 3. CE and combined sets demonstrate discordant interpretations of traumatic fracture at left femur in 56 -year-old woman with vaginal cancer. Initial diagnosis of reader 1 from CE set was pathologic fracture due to focal bone abnormality on T1-weighted images with enhancement at fracture margin, in which DWI is added to CE set, reader 1 changed diagnosis to traumatic fracture.

(A) Coronal T1-weighted and (B) axial contrast enhanced fat-saturated T1-weighted images show single bone abnormality with bone marrow enhancement at fracture site in left proximal femur, neck (arrows). (C) Corresponding axial DWI shows no high signal at fracture site $(b=1400$ $\mathrm{s} / \mathrm{mm}^{2}$ ). (D) Axial ADC map shows no impeded water diffusivity. This case was pathologically confirmed as intramedullary hemorrhage with traumatic fracture.

\section{Observer Reproducibility and Diagnostic Confidence}

Compared to the CE set, the combined set showed excellent inter-reader agreement, with a weighted $\kappa$ value of 0.830 (95\% confidence interval [CI]: 0.760-0.901). Inter-reader agreement was good in the CE and DW sets, with weighted $\kappa$ values of 0.665 (95\% CI: $0.574-0.757$ ) and 0.675 (95\% CI: 0.577-0.773), respectively. For both readers, the mean diagnostic confidence increased in the combined set compared to the CE set for traumatic fractures (R1, 2.66-2.84; R2, 2.65-2.72).

\section{DISCUSSION}

Our results showed that compared to the CE set, the combined set showed improved accuracy and specificity for diagnosis of pathologic fractures at extremities, while retaining sensitivity. This indicates the role of DWI when combined with conventional MRI: equivocal MRI findings in elderly patients who are likely to have underlying malignancy might lead to a false-positive diagnosis. Such incorrect diagnoses in the CE set could be corrected by confirming the 
absence of tumor using DWI and ADC, which was consistent with results in the previous studies $(5,14,15)$. These results could be advantageous for appropriately managing patients at risk by eliminating, or at least reducing, the false-positive diagnoses of pathologic fractures. This explanation also supports the improved confidence level in the traumatic fracture group after adding DWI.

We evaluated the ADC map qualitatively; however, its diagnostic performance in our study was similar to that in previous studies (sensitivity, $81-100 \%$; specificity, 78.9-97\%) (10,16). Absolute ADC values can be affected by many factors, such as scanner parameters and vendorspecific issues, including field inhomogeneity, coils, eddy currents, and sequence designs $(17,18)$. ADC value measurement involves additional confounding factors, such as region of interest (ROI) positioning, because the size and location of an ROI is decisive in ADC quantitative analysis, which necessitates avoiding artifacts, partial volume effects, and areas of hemorrhage and necrosis. Investigations regarding optimal positioning are ongoing despite reports on comparisons among different methods of ROI positioning $(19,20)$. We speculated that correlating the presumed solid portions on conventional MRI with DWI and ADC can provide sufficient guidance, leading to enhanced diagnostic accuracy without measuring ADCs. Pathologic fractures at extremities may demonstrate complex appearances, with hemorrhage, bone marrow of the fractured fragments, and intermingled adjacent soft tissue changes. Such situations can cause further challenges in accurate ROI positioning for representing the solid portion rather than vertebral compression fractures.

With conventional MRI, morphologic evaluation of pathologic fractures in cancer patients are based on the presence of focal bone abnormalities, indicating a tumor, extra-osseous soft tissue mass, or irregular areas of marked and inhomogeneous contrast enhancement of fractured bones (3). Multiple lesions can also suggest the presence of metastasis. In this study, we focused on the complicated situations that impede confident diagnoses of metastatic pathologic fractures in the clinical setting; for this purpose, we performed subgroup analyses excluding cases of multiple bone lesions, existing extra-osseous soft tissue component, or absent bone enhancement at fracture margins. In our study, pathologic fracture without an extra-osseous soft tissue mass was observed in up to $69.4 \%$ (34/49). Moreover, traumatic fracture showing bone enhancement at fracture margins was observed over $80 \%$ patients, although they had varying enhancement degrees. Bone marrow at the acute traumatic fracture site becomes edematous or is replaced by intramedullary hematomas and then shows a hyperemic response, thus altering the marrow relaxation time and showing fracture site enhancement $(4,8,11)$. In our study, the additional use of DWI with conventional MRI demonstrated improved accuracy and specificity, particularly for the less-experienced reader, in cases of absent extraosseous soft tissue mass and present bone enhancement, i.e., the situations that may make accurate interpretation more challenging on conventional MRI.

In this study, in cases of single bone abnormality, the combined set showed a trend of improvement in accuracy and specificity for reader 1, and up to $100 \%$ sensitivity, specificity and accuracy for reader 2, although the difference was not statistically significant. When a single bone abnormality is found in a patient who has a suspected pathologic fracture, it is not easy to distinguish benign from malignant causes, particularly when the size of a focal bone lesion is small without associated findings on MRI. Moreover, the presence of single-bone metastasis is considerably important as it may affect tumor stage in cancer patients. In such cases of our study, excluding the false-positive cases by demonstrating no impeded water diffusivity on DWI and ADC map could increase the specificity, and eventually increase the accuracy. Notably, there was a false positive case where DWI did not aid in establishing a correct diagnosis. Pathologically proven focal hematopoietic marrow in the femur which showed subtle high signal change on DWI and low signal intensity on ADC map was misdiagnosed as metastasis by reader 1 . This wellknown misleading focus on DWI and ADC map emphasizes the importance of fundamental analysis of morphologic features on conventional MRI.

CE imaging has become a key component of conventional MRI to discriminate underlying normal bone marrow from tumor involvement for assessing malignant pathologic fracture. However, moderate specificity of the CE set was observed in our study, and this was consistent with results of the previous studies, which reported limited specificity ( $71 \%$ and $70 \%$ ) of CE images in diagnosing vertebral compression fractures $(12,13)$. In our study, when comparisons were made between the CE and DW sets and between the DW and combined sets, the diagnostic performance did not differ significantly. Therefore, our results suggest that DWI could be an alternative imaging sequence to CE imaging for evaluating pathologic fractures 
in patients with impaired renal function or unstable conditions.

Our study has some limitations. First, it was a retrospective study with relatively small number of patients. Nonetheless, the cause of fractures was confirmed by biopsy or operation in more than $50 \%$ of patients. Furthermore, any study did not investigate whether and how the addition of DWI to conventional MRI improved diagnostic performance for differentiating pathologic and traumatic fractures at extremities. Second, we did not measure ADC values but instead focused on visual analysis, and thus, we could not propose cut-off reference values for differentiation. Drawing an ROI on a lesion could be confounded by many factors including limited reproducibility in quantitative analysis $(21,22)$, however, we believe that such qualitative analysis is preferable in clinical practice, particularly for evaluating pathologic fractures at extremities where often heterogeneous and complicated findings are observed. Third, in analyzing bone enhancement at fracture margins on conventional MRI, we did not evaluate the enhancement patterns and degrees, as enhancement might have varied at fractures over time. Time to healing is also variable, especially in elderly with potentially impeded reparative capacity. Fourth, we included patients with different kinds of primary cancers. Signal intensity of bone metastasis on DWI and ADC map may be different depending on cancer types, although no significant associations between ADC values and types of primary cancer were found for bone metastasis (23). Fifth, we included both myeloma and lymphoma cases. However, orthopedic management of their pathologic fractures is closely related to the treatment of the metastatic lesions.

In conclusion, combining DWI with conventional MRI improved diagnostic accuracy and specificity while retaining sensitivity in the evaluation of suspected pathologic fractures from metastasis at extremities, even in cases of absent extra-osseous soft tissue mass and present bone enhancement at fracture margins, which may make interpretation more challenging with conventional MRI, especially for less-experienced radiologists. DWI could be a useful supplement to routine MRI protocols for differentiating between pathologic and traumatic fractures at extremities from metastasis.

\section{Conflicts of Interest}

The authors have no potential conflicts of interest to disclose.
ORCID iDs

Min Hee Lee

https://orcid.org/0000-0001-9481-2138

Sun-Young Park

https://orcid.org/0000-0001-6500-3411

\section{REFERENCES}

1. Saad F, Lipton A, Cook R, Chen YM, Smith M, Coleman R. Pathologic fractures correlate with reduced survival in patients with malignant bone disease. Cancer 2007;110:18601867

2. Ward WG, Spang J, Howe D. Metastatic disease of the femur. Surgical management. Orthop Clin North Am 2000;31:633-645

3. Bae JH, Lee IS, Song YS, Kim JI, Cho KH, Lee SM, et al. Bone tumors with an associated pathologic fracture: differentiation between benign and malignant status using radiologic findings. J Korean Soc Radiol 2015;73:240-248

4. Jung HS, Jee WH, McCauley TR, Ha KY, Choi KH. Discrimination of metastatic from acute osteoporotic compression spinal fractures with MR imaging. Radiographics 2003;23:179-187

5. Baur A, Dietrich 0, Reiser M. Diffusion-weighted imaging of bone marrow: current status. Eur Radiol 2003;13:1699-1708

6. Padhani AR, van Ree K, Collins DJ, D'Sa S, Makris A. Assessing the relation between bone marrow signal intensity and apparent diffusion coefficient in diffusion-weighted MRI. AJR Am J Roentgenol 2013;200:163-170

7. Lee SY, Jee WH, Jung JY, Park MY, Kim SK, Jung CK, et al. Differentiation of malignant from benign soft tissue tumours: use of additive qualitative and quantitative diffusionweighted MR imaging to standard MR imaging at 3.0 T. Eur Radiol 2016;26:743-754

8. Zhou XJ, Leeds NE, McKinnon GC, Kumar AJ. Characterization of benign and metastatic vertebral compression fractures with quantitative diffusion MR imaging. AJNR Am J Neuroradiol 2002;23:165-170

9. Geith T, Schmidt G, Biffar A, Dietrich 0, Durr HR, Reiser M, et al. Comparison of qualitative and quantitative evaluation of diffusion-weighted MRI and chemical-shift imaging in the differentiation of benign and malignant vertebral body fractures. AJR Am J Roentgenol 2012;199:1083-1092

10. Sung JK, Jee WH, Jung JY, Choi M, Lee SY, Kim YH, et al. Differentiation of acute osteoporotic and malignant compression fractures of the spine: use of additive qualitative and quantitative axial diffusion-weighted MR imaging to conventional MR imaging at 3.0 T. Radiology 2014;271:488-498

11. Hamimi A, Kassab F, Kazkaz G. Osteoporotic or malignant vertebral fracture? This is the question. What can we do about it? The Egyptian Journal of Radiology and Nuclear Medicine 2015;46:97-103

12. Bhugaloo A, Abdullah B, Siow Y, Ng K. Diffusion weighted MR imaging in acute vertebral compression fractures: differentiation between malignant and benign causes. Biomed 
Imaging Interv $\mathrm{J}$ 2006;2:e12

13. Abowarda MH, Abdel-Rahman HM, Taha MM. Differentiation of acute osteoporotic from malignant vertebral compression fractures with conventional MRI and diffusion MR imaging.

The Egyptian Journal of Radiology and Nuclear Medicine 2017;48:207-213

14. Del Grande F, Subhawong T, Weber K, Aro M, Mugera C, Fayad LM. Detection of soft-tissue sarcoma recurrence: added value of functional MR imaging techniques at 3.0 T. Radiology 2014;271:499-511

15. Kim SH, Lee JM, Hong SH, Kim GH, Lee JY, Han JK, et al. Locally advanced rectal cancer: added value of diffusionweighted MR imaging in the evaluation of tumor response to neoadjuvant chemo- and radiation therapy. Radiology 2009;253:116-125

16. Park HJ, Lee SY, Rho MH, Chung EC, Kim MS, Kwon HJ, et al. Single-shot echo-planar diffusion-weighted MR imaging at $3 T$ and 1.5T for differentiation of benign vertebral fracture edema and tumor infiltration. Korean J Radiol 2016;17:590-597

17. Kivrak AS, Paksoy Y, Erol C, Koplay M, Özbek S, Kara F. Comparison of apparent diffusion coefficient values among different MRI platforms: a multicenter phantom study. Diagn Interv Radiol 2013;19:433-437

18. Sasaki M, Yamada K, Watanabe Y, Matsui M, Ida M, Fujiwara $S$, et al. Variability in absolute apparent diffusion coefficient values across different platforms may be substantial: a multivendor, multi-institutional comparison study. Radiology
2008;249:624-630

19. Blazic IM, Lilic GB, Gajic MM. Quantitative assessment of rectal cancer response to neoadjuvant combined chemotherapy and radiation therapy: comparison of three methods of positioning region of interest for $A D C$ measurements at diffusion-weighted MR imaging. Radiology 2017;282:615

20. Ahlawat S, Khandheria P, Del Grande F, Morelli J, Subhawong TK, Demehri S, et al. Interobserver variability of selective region-of-interest measurement protocols for quantitative diffusion weighted imaging in soft tissue masses: comparison with whole tumor volume measurements. J Magn Reson Imaging 2016;43:446-454

21. Braithwaite AC, Dale BM, Boll DT, Merkle EM. Short- and midterm reproducibility of apparent diffusion coefficient measurements at 3.0-T diffusion-weighted imaging of the abdomen. Radiology 2009;250:459-465

22. Kim SY, Lee SS, Byun JH, Park SH, Kim JK, Park B, et al. Malignant hepatic tumors: short-term reproducibility of apparent diffusion coefficients with breath-hold and respiratory-triggered diffusion-weighted MR imaging. Radiology 2010;255:815-823

23. Cha MJ, Yoon YC. Clinical relevance of the apparent diffusion coefficient value of metastatic bone tumours on diffusionweighted MRI images: differences according to the types of primary tumour, the affected bones, and clinical factors. Clin Radiol 2015;70:1116-1121 